\title{
Role of Proton Diffusion in the Nonexponential Kinetics of Proton-Coupled Electron Transfer from Photoreduced ZnO Nanocrystals
}

\author{
Soumya Ghosh, Alexander V. Soudackov, and Sharon Hammes-Schiffer*il
}

Department of Chemistry, 600 South Mathews Avenue, University of Illinois at Urbana-Champaign, Urbana, Illinois 61801, United States

Supporting Information

ABSTRACT: Experiments have suggested that photoreduced $\mathrm{ZnO}$ nanocrystals transfer an electron and a proton to organic radicals through a concerted proton-coupled electron transfer (PCET) mechanism. The kinetics of this process was studied by monitoring the decay of the absorbance that reflects the concentration of electrons in the conduction bands of the nanocrystals. Interestingly, this absorbance exhibited nonexponential decay kinetics that could not be explained by heterogeneities of the nanoparticles or electron content. To determine if proton diffusion from inside the nanocrystal to reactive sites on the surface could lead to such nonexponential kinetics, herein this process is modeled using kinetic Monte Carlo simulations. These simulations provide the survival probability of a proton hopping among bulk, subsurface, and surface sites within the nanocrystal until it reaches a reactive surface site where it

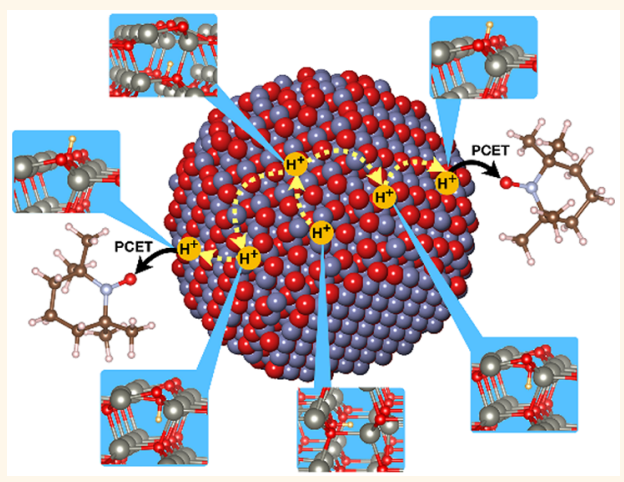
transfers to an organic radical. Using activation barriers predominantly obtained from periodic density functional theory, the simulations reproduce the nonexponential decay kinetics. This nonexponential behavior is found to arise from the broad distribution of lifetimes caused by different types of subsurface and surface sites. The longer lifetimes are associated with the proton becoming temporarily trapped in a subsurface site that does not have direct access to a reactive surface site due to capping ligands. These calculations suggest that movement of the protons rather than the electrons dominate the nonexponential kinetics of the PCET reaction. Thus, the impact of both bulk and surface properties of metal-oxide nanoparticles on proton conductivity should be considered when designing heterogeneous catalysts.

KEYWORDS: proton-coupled electron transfer, proton diffusion, proton conductivity, nanocrystal, nanoparticle, kinetic Monte Carlo, density functional theory

$\mathrm{M}$ etal-oxide nanoparticles have been extensively studied both experimentally and theoretically as electron-transfer agents, predominantly in relation to their potential applications in dye-sensitized solar cells. ${ }^{1-5}$ Although the majority of these studies have focused on $\mathrm{TiO}_{2}$, $\mathrm{ZnO}$ nanomaterials have also generated widespread attention due to their optical properties, thermal stability, and high specific surface area, which is a key factor for dye adsorption. ${ }^{8,9}$ In contrast, experimental studies of proton transfer and protoncoupled electron transfer (PCET) in metal-oxide nanoparticles are rare. Understanding the role of protons in these systems, however, is crucial for the design of catalysts to facilitate important transformations such as water oxidation, dioxygen reduction, $\mathrm{CO}_{2}$ to methanol conversion, $\mathrm{C}-\mathrm{H}$ activation, dinitrogen reduction, and a variety of other transformations involving the net transfer of multiple electrons and protons. $^{10-12}$

In recent studies, ${ }^{13}$ photoreduced $\mathrm{ZnO}$ nanoparticles have been prepared by photogeneration of electron/hole pairs, followed by quenching of the holes through oxidation of ethanol, leaving electrons in the conduction band $(\mathrm{CB})$ and protons at the surface and in the bulk. These photoreduced $\mathrm{ZnO}$ nanoparticles have been shown to undergo PCET reactions with organic radicals such as TEMPO and ${ }^{t} \mathrm{Bu}_{3} \mathrm{ArO}$ based on flash photolysis experiments. ${ }^{13}$ In these experiments, the photoreduced nanocrystal (NC), which is denoted $\mathrm{ZnO}: e_{\mathrm{CB}}^{-} / \mathrm{H}^{+}$to indicate that the reducing electrons occupy delocalized orbitals in the $\mathrm{CB}$, transfers an electron and a proton to an organic radical such as TEMPO to produce $\mathrm{ZnO}$ and TEMPOH. The reaction between photoreduced $\mathrm{ZnO}$ NCs and TEMPO has been further studied using stopped-flow kinetics, providing information about the kinetics on longer time scales $(\sim 2 s) .{ }^{14}$ In these experiments, the PCET reaction

Received: July 16, 2017

Accepted: September 19, 2017

Published: September 19, 2017 
was studied by monitoring the absorbance at $700 \mathrm{~nm}$, which scales linearly with the concentration of electrons in the CBs of the NCs, and the concentration of TEMPO was significantly greater than the effective concentration of the photoreduced NC. These experimental conditions are expected to yield pseudofirst-order kinetics, but surprisingly, the measured decay of absorbance at $700 \mathrm{~nm}$, which reflects the decrease in the concentration of $\mathrm{CB}$ electrons, exhibited nonexponential kinetics.

Several possible hypotheses have been proposed to explain the deviation from exponential decay kinetics for PCET from photoreduced $\mathrm{ZnO}$ NCs to TEMPO. One plausible hypothesis is that the NC size distribution of $\sim 17 \%$ about the mean is accompanied by a distribution of electron content that leads to different electron-transfer rates. This hypothesis was discarded because NC solutions with similar concentrations of $\mathrm{CB}$ electrons displayed different reactivity, and previous studies of electron transfer from $\mathrm{PbS} \mathrm{NCs}$ to quinones, with similarly broad NC size distributions, displayed single exponential kinetic behavior. ${ }^{15}$ The deviation from exponential decay kinetics is also thought to be too significant to be explained by variations in the size and shape of the NCs for a size distribution of $\pm 17 \%$ or by the heterogeneity in the capping group number and density. After discarding these possible explanations, a remaining possible explanation is that the nonexponential kinetics arises from the behavior of the protons that participate in the PCET reaction to TEMPO. ${ }^{14}$

The goal of the current work is to investigate whether diffusion of protons from inside the NC to reactive sites on the NC surface can lead to the observed nonexponential kinetic behavior. Previous extensive theoretical and experimental studies suggest that protons can intercalate into bulk $\mathrm{ZnO}$ and contribute to n-type conductivity. ${ }^{16-23}$ In addition, hightemperature annealing behavior of hydrogen-doped $\mathrm{ZnO}$ has been explained in terms of hydrogen diffusion from interstitial and oxygen vacancy sites. ${ }^{24-26}$ Moreover, hydrogen on $\mathrm{Zn}$ sites of the nonpolar $10 \overline{1} 0$ surface of wurtzite crystal has been proposed to diffuse inside the bulk at temperatures above 200 $\mathrm{K}^{27}$ In this study, we employ a plausible model for the $\mathrm{ZnO}$ NC with a specified concentration of reactive sites on the surface. A single proton starts from a lattice site inside the NC and moves stochastically between sites, accounting for relevant barriers between different types of sites, until it reaches one of the reactive sites where it can be transferred to TEMPO. The barriers between different types of sites are calculated with periodic density functional theory (DFT), and the survival probability of the proton, which is defined in terms of the time required to reach a reactive site, is computed within the framework of a kinetic Monte Carlo (KMC) scheme. ${ }^{28,29}$ The calculated survival probability is compared to the experimentally measured normalized absorbance versus time, and the results are analyzed in terms of the physical properties of the NCs.

\section{RESULTS AND DISCUSSION}

Model for $\mathrm{ZnO}$ NC. A model for the $\mathrm{ZnO} \mathrm{NC}$ was designed by carving out a nanoparticle of radius $2 \mathrm{~nm}$ centered at a $\mathrm{Zn}$ atom within the wurtzite lattice, as depicted in Figure 1. The $\mathrm{H}$ sites in this NC are subdivided into three categories, namely surface sites, subsurface sites, and bulk sites, based on geometric criteria. All $\mathrm{O}(\mathrm{Zn})$ atoms with fewer than four nearestneighbor $\mathrm{Zn}(\mathrm{O})$ atoms are considered to be surface atoms. In our model, $255 \mathrm{O}$ atoms belong to the nonpolar surfaces, and

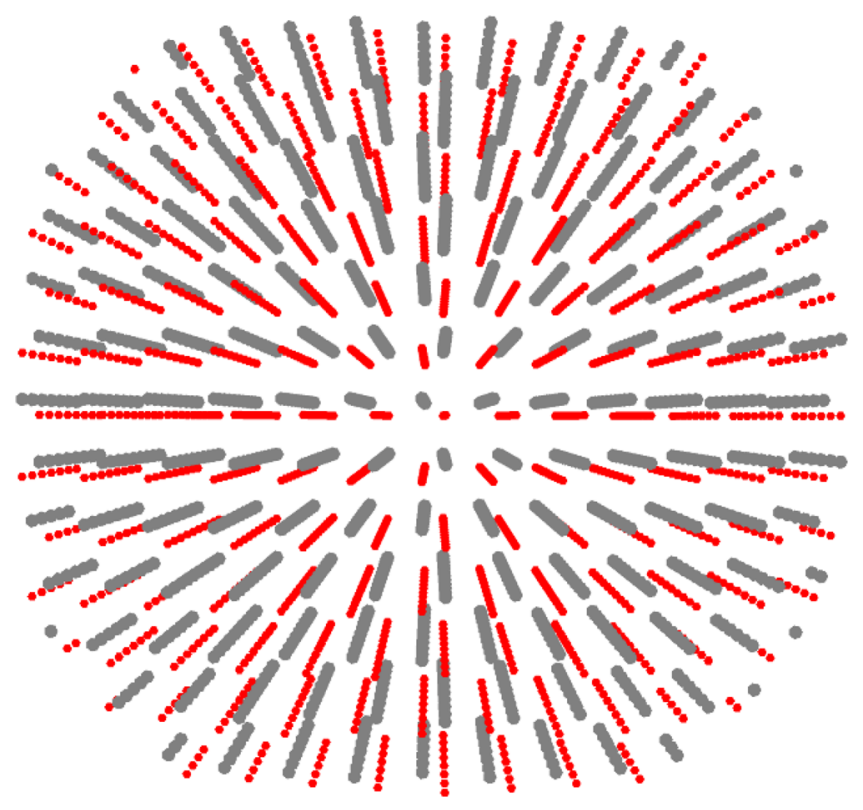

Figure 1. Model for the $\mathrm{ZnO} \mathrm{NC}$ used in the KMC simulations.

$48 \mathrm{O}$ atoms belong to the polar surface. The polar $\mathrm{O}$ sites are assumed to be continuously occupied with protons because of the large hydrogen atom dissociation energy associated with these sites and therefore are not considered as potential proton hopping sites. The definitions of subsurface and bulk sites are given below in Theoretical Methods. The relevant $\mathrm{H}$ sites, which correspond to the proton bonded to an $\mathrm{O}$ atom, are shown in Figure 2. A specified fraction of the "surface:up" sites is assumed to be reactive for PCET to TEMPO, while the remaining surface sites are assumed to be blocked with unreactive protons because the capping ligands prevent TEMPO from approaching close enough to allow PCET.

In the KMC simulations, a proton starting from a bulk site or a subsurface site performs a random walk based on microscopic rate constants for hopping between sites until it reaches a reactive site on the surface, where it can undergo a fast PCET reaction. The activation barriers for proton hopping between different sites were calculated with DFT and are provided in Table 1. In these calculations, a neutral hydrogen atom was inserted into the NC. An interstitial hydrogen atom in $\mathrm{ZnO}$ is known to be a shallow donor with its electronic state almost isoenergetic with the lower $\mathrm{CB}$ edge of $\mathrm{ZnO} .^{16-19}$ Thus, an electron is thought to be donated to one of the delocalized states near the $\mathrm{CB}$ edge, and diffusion of hydrogen in $\mathrm{ZnO}$ can be described as diffusion of a positively charged proton. A proton in a bulk site can hop to another bulk site or to a subsurface site. A proton in a subsurface site can hop to another subsurface site, to a surface:down site, or to a bulk site. A proton in a surface:down site can rotate to a surface:up site or can hop to a subsurface site. A proton in a surface:up site can rotate to a surface:down site or hop to another surface:up site. The surface:down site corresponding to a blocked surface:up site is assumed to be inaccessible to any proton in the subsurface layer because a given oxygen atom is presumed to be bonded to a single proton. The barrier for hopping between a bulk and subsurface site, $E_{\mathrm{a}}^{\mathrm{B} \rightarrow \mathrm{S}}$, is treated as a parameter because it could not be calculated in a straightforward manner. More details about these calculations are provided below in Theoretical Methods. 
A

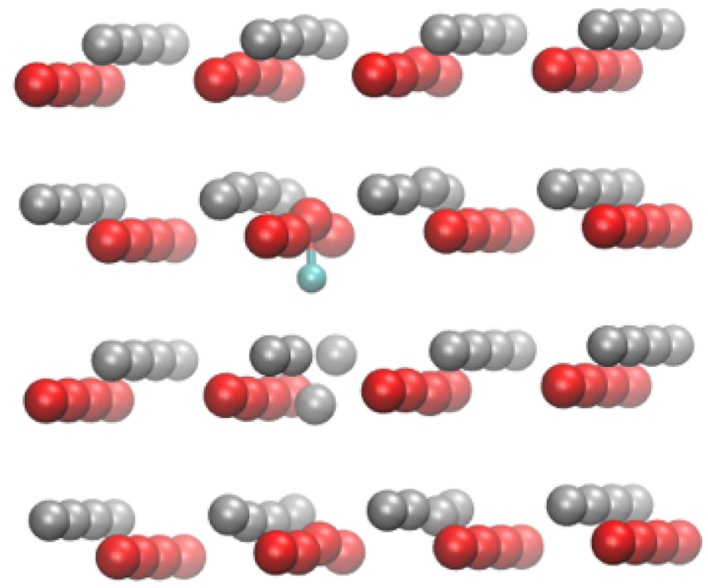

C
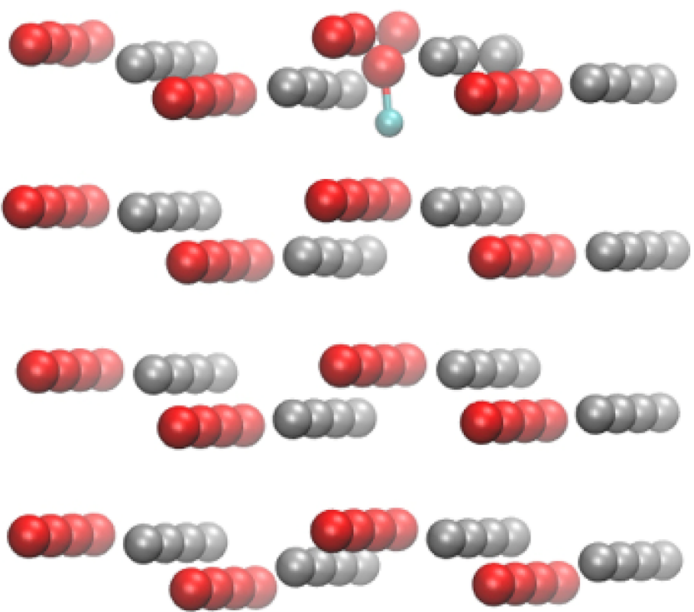

B
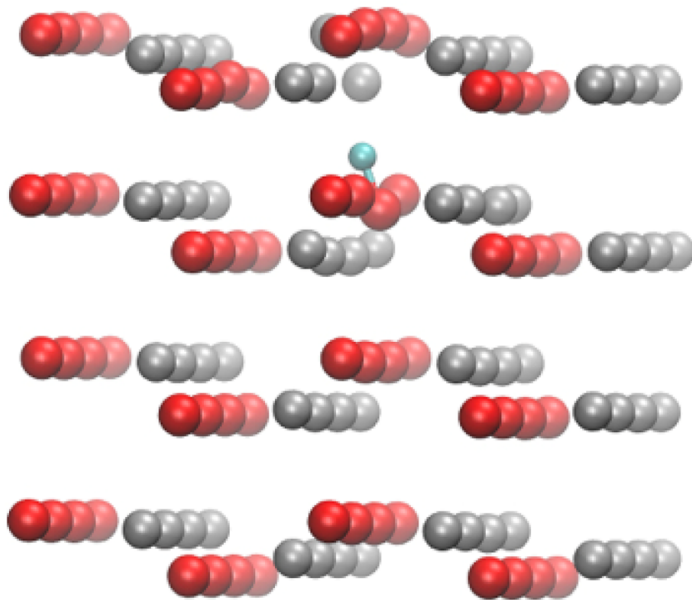

D
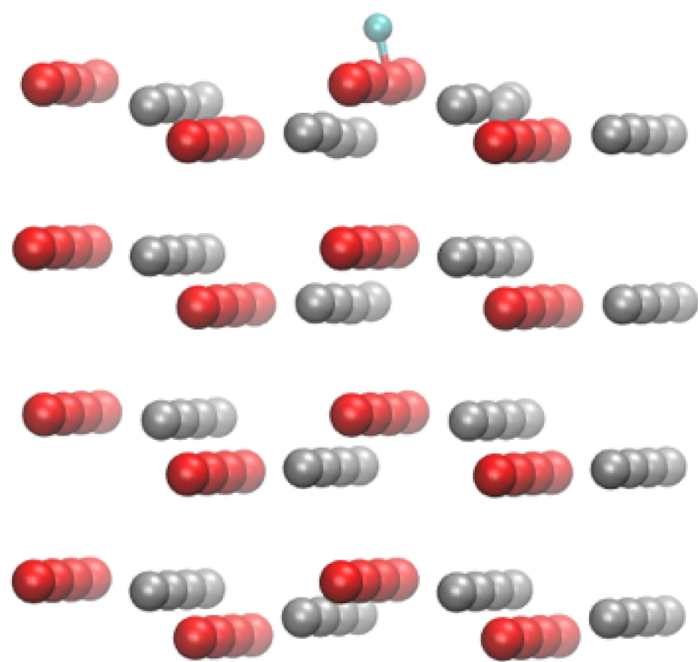

Figure 2. $\mathrm{H}$ sites within the $\mathrm{ZnO} \mathrm{NC}$ with the active proton shown in cyan: (A) $\mathrm{BC}_{\|}$bulk, (B) subsurface, (C) surface:down, and (D) surface:up sites. Here $\mathrm{BC}_{\|}$denotes bond-centered (i.e., in between an $\mathrm{O}$ and $\mathrm{Zn}$ atom) along the c-axis of the wurtzite crystal.

Table 1. Activation Energies (in eV) for Proton Hopping from the Site Specified in the Row to the Site Specified in the Column ${ }^{a}$

$\begin{array}{lcccl} & \text { surface:up } & \text { surface:down } & \text { subsurface } & \text { bulk } \\ \text { surface:up } & 1.2 & 0.9 & \mathrm{~N} / \mathrm{A} & \mathrm{N} / \mathrm{A} \\ \text { surface:down } & 0.3 & \mathrm{~N} / \mathrm{A} & 0.2 & \mathrm{~N} / \mathrm{A} \\ \text { subsurface } & \mathrm{N} / \mathrm{A} & 0.3 & 1.2 & E_{a}^{\mathrm{B} \rightarrow \mathrm{S}} \\ \text { bulk } & \mathrm{N} / \mathrm{A} & \mathrm{N} / \mathrm{A} & E_{a}^{\mathrm{B} \rightarrow \mathrm{S}} & 0.7\end{array}$

${ }^{a} \mathrm{~N} / \mathrm{A}$ indicates that the hop was not allowed in the KMC simulations, and $E_{a}^{\mathrm{B} \rightarrow \mathrm{S}}$ indicates that this activation energy was treated as a parameter in the KMC simulations. A hop between two surface:down sites is not allowed because it would go through a subsurface site.

Analysis of Kinetics for Photoreduced ZnO NCs. In our model, the kinetics of the overall process is dictated predominantly by proton diffusion inside the bulk of the NC because the PCET reaction at the surface occurs on a much faster time scale $\left(\sim 1000 \mathrm{~s}^{-1}\right)$ compared to hopping between two bulk sites $\left(\sim 6.6 \mathrm{~s}^{-1}\right)$. We analyze the overall kinetics in terms of the time dependence of the survival probability, defined to be the fraction of random walks for which the proton has not yet reacted. As shown in Figure 3, the calculated survival probability versus time is in excellent agreement with the experimentally measured time decay of the normalized absorbance of photoreduced $\mathrm{ZnO} \mathrm{NCs}$ in the presence of TEMPO if the two free parameters are chosen appropriately.

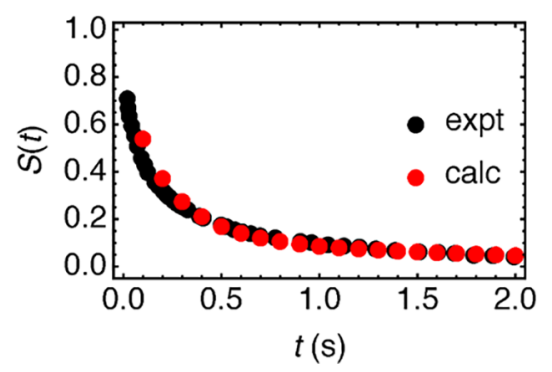

Figure 3. Comparison of the experimental kinetics data (black) and the time dependence of the survival probability calculated with the KMC simulations (red) with an activation barrier between a bulk site and a subsurface site of $0.8 \mathrm{eV}$ and $70 \%$ of the surface sites reactive. The experimental data were obtained from Figure $3 b$ (blue curve) in ref 14 . 
First, $70 \%$ of surface sites are assumed to be reactive, and second, $E_{\mathrm{a}}^{\mathrm{B} \rightarrow \mathrm{S}}$ is chosen to be $0.8 \mathrm{eV}$, which is $0.1 \mathrm{eV}$ larger than the activation barrier for hopping between two bulk sites. For the remainder of this section, we analyze how changing both the fraction of reactive surface sites and $E_{a}^{\mathrm{B} \rightarrow \mathrm{S}}$ impacts the survival probability, and we provide physical justification for the choices.

The concentration of reactive sites on the surface of the NC depends on the concentration of the capping ligands. The calculated survival probability at a particular time decreases as the concentration of the reactive sites on the surface increases (Figure S1). Experimentally, $\sim 25 \%$ of the surface $\mathrm{Zn}$ sites are bound to organic capping ligands. ${ }^{30}$ Protonated $\mathrm{O}$ sites that are accessible to TEMPO, despite these capping ligands, can react with TEMPO by way of PCET. In our simulations, $\sim 70 \%$ of the $\mathrm{O}$ atoms of the nonpolar surfaces are assumed to be reactive, while the remaining unreactive $\mathrm{O}$ atoms are assumed to be protonated on the outer surface. This concentration of reactive sites is qualitatively reasonable considering that $25 \%$ of the surface $\mathrm{Zn}$ sites are capped with ligands that would block TEMPO from approaching.

The different values of $E_{\mathrm{a}}^{\mathrm{B} \rightarrow \mathrm{S}}$ considered in comparison to the other relevant activation barriers are depicted in Figure 4.
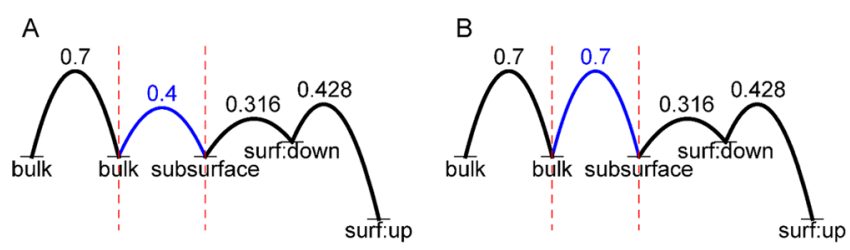

C
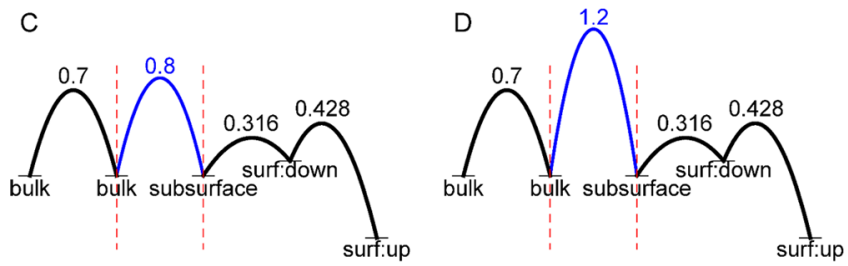

Figure 4. Relative energy diagrams illustrating the key activation barriers for proton diffusion in the $\mathrm{ZnO}$ NCs. The activation barrier for proton hopping between a bulk site and a subsurface site (blue) is varied from parts $A$ to $D$. All activation barriers are given in eV. Here surf:down and surf:up correspond to surface:down and surface:up, respectively.

When $E_{\mathrm{a}}^{\mathrm{B} \rightarrow \mathrm{S}}=0.4 \mathrm{eV}$, the survival probability decays exponentially (Figure 5), whereas when $E_{\mathrm{a}}^{\mathrm{B} \rightarrow \mathrm{S}}=1.2 \mathrm{eV}$, the decay is best represented by a biexponential function (Figure 5). The survival probabilities calculated with bulk to subsurface activation barriers of $E_{\mathrm{a}}^{\mathrm{B} \rightarrow \mathrm{S}}=0.7 \mathrm{eV}$ and $E_{\mathrm{a}}^{\mathrm{B} \rightarrow \mathrm{S}}=0.8 \mathrm{eV}$ can be fit well with both biexponential and stretched exponential functions (Figure 5). These results invoke two major questions. First, why does the time dependence of the survival probability deviate from single exponential kinetics upon increasing the activation barrier for hopping between bulk and subsurface sites from 0.4 to $0.8 \mathrm{eV}$ and beyond? Second, why does the stretching parameter decrease from 0.80 to 0.54 when $E_{\mathrm{a}}^{\mathrm{B} \rightarrow \mathrm{S}}$ is increased from 0.7 to $0.8 \mathrm{eV}$ ? Critical to understanding these two questions are the relative barriers for proton hopping between different sites, as summarized in Figure 4, and the inaccessibility of the surface:down sites associated with blocked surface:up sites.
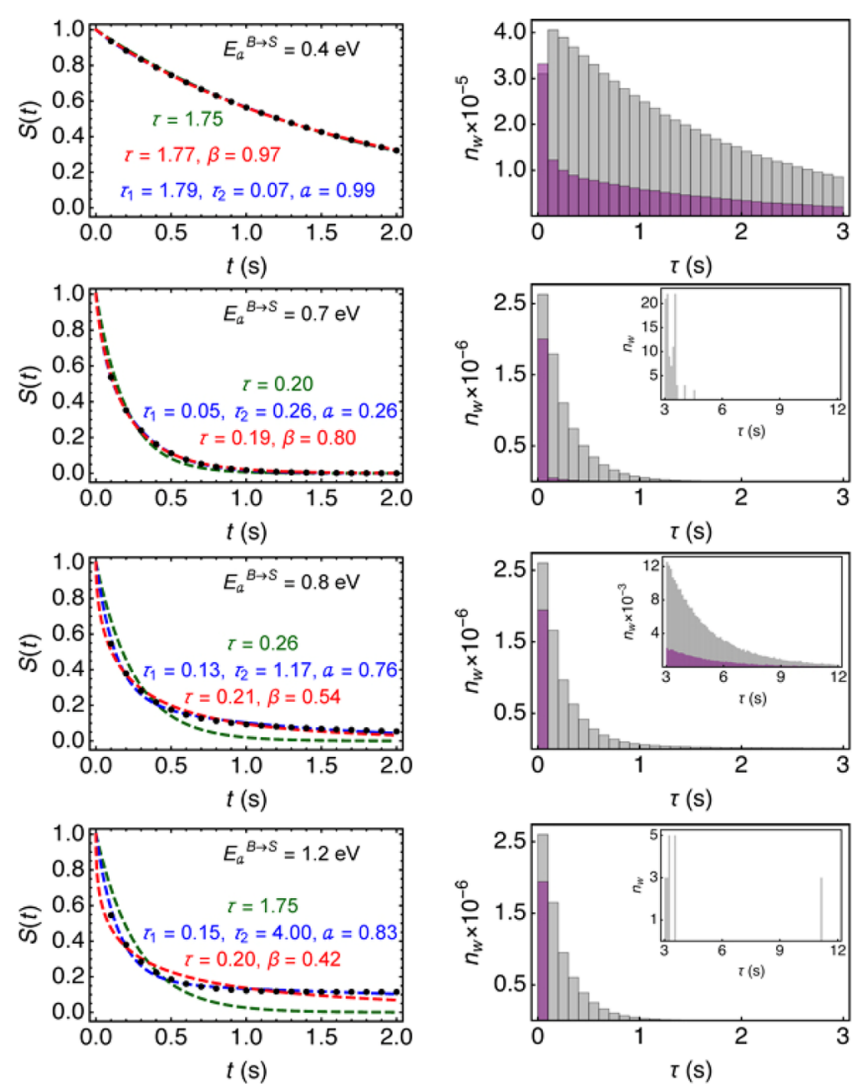

Figure 5. Calculated survival probabilities $S(t)$ and corresponding histograms for lifetimes of walks, where $n_{w}(\tau)$ is the number of walks with lifetime $\tau$. Starting from the top, the rows correspond to $E_{\mathrm{a}}^{B \rightarrow S}=0.4,0.7,0.8$, and $1.2 \mathrm{eV}$. On the left, the black circles represent data from the KMC simulations, and the lines represent fits to these data: single exponential (green: $\exp [-t / \tau]$ ), biexponential (blue: $\left.a \exp \left[-t / \tau_{1}\right]+(1-a) \exp \left[-t / \tau_{2}\right]\right)$, and stretched exponential (red: $\exp \left[-(t / \tau)^{\beta}\right]$ ). On the right, the gray and purple bars correspond to walks starting at a bulk site or a subsurface site, respectively. The insets show a magnification of the histograms at longer time scales, as indicated by the $y$-axis units. According to eq $1, S(t)$ is the integral of $n_{w}(\tau)$ for $\tau=t$ to $\infty$.

To analyze the survival probabilities, we plotted the histograms of the lifetimes for all of the random walks (Figure $5)$. The histograms of the lifetimes are directly related to the corresponding survival probabilities $S(t)$ :

$$
S(t)=\frac{N_{\mathrm{w}}(t)}{N}=\frac{1}{N} \int_{t}^{\infty} n_{\mathrm{w}}(\tau) d \tau
$$

where $N_{\mathrm{w}}(t)$ is the total number of walks that have lifetimes $\geq t$, $N$ is the total number of walks, and $n_{\mathrm{w}}(\tau)$ is the number of walks with lifetime $\tau$. According to eq 1 , if the histogram of lifetimes shows exponential decay, then the survival probability will also show exponential decay kinetics. Alternatively, if the histogram becomes nonexponential (multiexponential or stretched exponential), then the survival probability will also exhibit similar characteristics. All of the walks are subdivided into walks that originated either from the subsurface sites (purple bars in Figure 5) or from the bulk sites (gray bars in Figure 5). The total number of walks starting from either bulk or subsurface sites is proportional to the total number of available bulk or subsurface sites, respectively. In all cases, the overall survival probability is dominated by the decay kinetics of the histogram of walks originating from bulk sites because the 
number of bulk sites is significantly larger than the number of subsurface sites, and the histograms for walks originating from the subsurface sites decay on a much faster time scale. Hence, we interpret the decay kinetics of the overall survival probability in terms of the histograms for walks originating from the bulk sites only.

When the activation barrier for proton hopping between a bulk site and a subsurface site is much smaller than the corresponding barrier connecting two bulk sites (Figure 4A), the histogram for walks originating from bulk sites decays exponentially (Figure 5, top row). In this case, the activation barrier for proton hopping from a bulk site to a subsurface site is similar to that for proton hopping from a subsurface site to a surface site (Figure 4A). This similarity in activation barrier translates to a comparable probability of hopping from a subsurface site to either a bulk site or a surface site where it can react. Even when the neighboring surface site of a subsurface site is inaccessible, the proton can still hop to a neighboring bulk site and then find another subsurface site with access to a surface site. Thus, the proton can still react, and none of the walks becomes stuck beneath the surface. This situation is similar to free diffusion with a low concentration of randomly distributed static traps (reactive sites), where the solution to the reaction-diffusion equation shows effectively exponential decay of the survival probability. ${ }^{31}$

When the activation barrier for proton hopping between a bulk and a subsurface site is increased to $0.7 \mathrm{eV}$ (Figure 4B), the probability of hopping from bulk to subsurface becomes comparable to the probability of hopping from bulk to bulk. On the other hand, the probability of hopping from a subsurface site to a bulk site decreases, leading to an effective increase in the probability of hopping from a subsurface site to a surface site where the reaction can occur. This combination of probabilities results in faster exponential decay kinetics observed at earlier times and accumulation of walks with intermediate lifetimes as shown in Figure 5 (second row), where the inset shows the histograms for the lifetimes at intermediate times. In addition, some of the walks survive for even longer times because the proton might get stuck in one of the subsurface sites without access to a reactive surface site. These features contribute to the slightly nonexponential behavior of the survival probability (Figure 5, second row). Upon further increasing the activation barrier for hopping between a bulk site and a subsurface site to be greater than the activation barrier for hopping between two bulk sites (Figure $4 \mathrm{C})$, hopping between two bulk sites is more probable than hopping from a bulk to a subsurface site, resulting in even more walks with intermediate lifetimes, as shown in the inset of the third row of Figure 5. Note that the nonexponential behavior of the survival probability is more pronounced for $E_{\mathrm{a}}^{\mathrm{B} \rightarrow \mathrm{S}}=0.8 \mathrm{eV}$ than for $E_{\mathrm{a}}^{\mathrm{B} \rightarrow \mathrm{S}}=0.7 \mathrm{eV}$ because of the greater relative probability for hopping between bulk sites.

When the activation barrier for proton hopping between a bulk site and a subsurface site is much larger than the corresponding barrier connecting two bulk sites (Figure 4D), the histogram for lifetimes between 0 and $2 \mathrm{~s}$ is similar to the cases with bulk to subsurface activation barriers of 0.7 and 0.8 $\mathrm{eV}$. In contrast to these two cases, however, the walks with intermediate lifetimes $(1.5 \mathrm{~s}<\tau<100 \mathrm{~s})$ become scarce (Figure 5, bottom row) because the probability of an indirect route connecting a subsurface site and a reactive surface site via a bulk site decreases dramatically. In addition, the walks with very long lifetimes $\left(>10^{4} \mathrm{~s}\right)$ increase in number. The resulting distribution of lifetimes exhibits a bimodal character, leading to approximately biexponential decay kinetics of the survival probability (Figure 5, bottom row).

Thus, the physical basis for the nonexponential kinetics is that the proton samples different types of sites, leading to different routes from the bulk to the surface with varying probabilities. In addition to distinct barriers for hopping between different types of sites, the presence of capping ligands results in a significant fraction of inaccessible surface sites with bound, nonreactive protons and leads to two different types of subsurface sites. These subsurface sites differ in whether they are adjacent either to a surface site that is accessible and therefore reactive or to a surface site that is inaccessible because a proton is already bound to it on the outer surface. As shown previously, ${ }^{32}$ stretched exponential behavior is expected for atomic diffusion with a broad distribution of lifetimes. For proton diffusion in these $\mathrm{ZnO}$ nanoparticles, two important properties are required to obtain the type of distribution that leads to the experimentally observed stretched exponential kinetics. First, there should be a low but non-negligible probability of the proton finding an alternate route from a subsurface site to a reactive surface site via a bulk site. Second, there should be a higher probability of hopping from a bulk site to another bulk site than to a subsurface site. When the activation barrier for proton hopping between two bulk sites is $0.7 \mathrm{eV}$, both of these properties are satisfied when the activation barrier for proton hopping from a bulk site to a subsurface site is $0.8 \mathrm{eV}$ (Figure $4 \mathrm{C}$ ). When the activation barrier for proton hopping from a bulk to a subsurface site is $0.7 \mathrm{eV}$ (Figure 4B), the second property is not satisfied. For an activation barrier of $1.2 \mathrm{eV}$ (Figure 4D), the first property is not satisfied, and for an activation barrier of $0.4 \mathrm{eV}$ (Figure 4A), neither property is satisfied. When the activation barrier for proton hopping from a bulk to a subsurface site is relatively large (i.e., $1.2 \mathrm{eV}$ ), the kinetics can also be described as biexponential decay. This biexponential behavior could be viewed in terms of two types of walks: those for which the proton becomes stuck in a subsurface site with an inaccessible neighboring surface site, and those for which the proton does not sample these types of subsurface sites and therefore reaches a reactive surface site more quickly.

We also investigated the sensitivity of the decay of the survival probability to the activation barrier for hopping between bulk sites. If this effective barrier is artificially increased to $0.8 \mathrm{eV}$, the time scale for the decay of survival probability changes from $\sim 2 \mathrm{~s}$ to $\sim 100 \mathrm{~s}$, but the stretching parameter $\beta$ for the stretched exponential decay still depends on the difference between the activation barriers for hopping between bulk sites and hopping from a bulk site to a subsurface site (see Figure S2).

Thus, we found that the experimental data could only be reproduced with this model if the activation barrier for proton hopping between a bulk site and a subsurface site is slightly greater than the activation barrier for proton hopping between two bulk sites. A possible physical explanation for this finding is that the bulk sites and subsurface sites are structurally different, and the resulting mismatch leads to a slightly longer effective hopping length between a bulk and a subsurface site than between two bulk sites, thereby potentially leading to a larger activation barrier. Another possible explanation is the presence of oxygen vacancies that are occupied by protons. Previous work has shown that a proton or a hydrogen atom at an oxygen vacancy is quite stable both thermodynamically and kineti- 
cally. ${ }^{25,26}$ If a large percentage $(\sim 70 \%)$ of regular bulk sites that have at least one subsurface site as a neighbor are replaced by oxygen vacancies occupied by protons, then the survival probability exhibits stretched exponential character with a slightly longer time scale and larger stretching parameter than experimentally observed (see Figure S3). It is possible that a combination of factors, including structural deformation, oxygen vacancies, excess protons, and organic capping ligands, contribute toward the experimentally observed stretching parameter.

\section{CONCLUSIONS AND IMPLICATIONS}

These calculations provide a plausible explanation for the origin of the experimentally measured nonexponential decay kinetics for the PCET reaction from photoreduced $\mathrm{ZnO} \mathrm{NCs}$ to TEMPO. The kinetics was determined experimentally by monitoring the decay of the absorbance at $700 \mathrm{~nm}$, which reflects the concentration of electrons in the CBs of the NCs. We modeled this kinetics using KMC simulations to monitor the decay of the survival probability of a proton hopping among bulk, subsurface, and surface sites within the NC. In these simulations, only a certain fraction of surface sites are accessible and reactive for PCET to TEMPO. Our calculations also indicate that an activation barrier of $0.7 \mathrm{eV}$ for proton hopping between two bulk sites provides the appropriate time scale for the decay. Most importantly, the simulations show that an activation barrier between bulk and subsurface sites of $0.8 \mathrm{eV}$ leads to excellent agreement with the experimentally measured nonexponential kinetics. The nonexponential kinetics is found to arise from the broad distribution of lifetimes caused by different types of subsurface and surface sites. In particular, the longer lifetimes are associated with the proton becoming temporarily trapped in a subsurface site that does not have direct access to a reactive surface site.

This work implies that the nonexponential kinetics of PCET reactions at the interfaces of metal-oxide nanoparticles is dominated by movement of the protons rather than the electrons and that proton diffusion may play an important role in the catalytic performance of such nanoparticles. In particular, the kinetics on longer time scales is found to be determined primarily by proton diffusion within the bulk, rather than only on the surface. Thus, both bulk and surface properties of metaloxide nanoparticles, especially in the context of proton conductivity, should be considered when designing heterogeneous catalysts that require PCET steps. These KMC simulations focused on proton diffusion to reactive surface sites but did not examine the PCET reaction occurring after the reactive site is reached. In recent work, we used vibronically nonadiabatic PCET theory to examine the PCET reaction from photoreduced $\mathrm{ZnO}$ NCs to TEMPO. ${ }^{33}$ The combined insights about both proton diffusion and the PCET mechanism could have significant implications for future catalyst design.

\section{THEORETICAL METHODS}

A nanoparticle of radius $2 \mathrm{~nm}^{14}$ centered at a $\mathrm{Zn}$ atom was carved out from the wurtzite lattice. To generate NCs that expose well-defined crystal planes at the surface while keeping an overall near-spherical shape, four symmetry equivalent nonpolar and two polar surfaces of the hexagonal wurtzite unit were generated for the $(n-1)^{\text {th }}$ layer of atoms, where $n$ denotes the outermost bilayer. All atoms of the nanoparticle within the region bounded by these planes constitute our model for the NC, which is depicted in Figure 1. The above steps ensure the following properties of the NC: dangling bonds on the surface are minimized, both polar and nonpolar surfaces are exposed, ${ }^{34}$ and a similar number of $\mathrm{Zn}$ and $\mathrm{O}$ atoms, 1309 and 1329, respectively, are included in the NC. The coordinates of all atoms in the NC are provided in the SI.

To determine whether it is possible to abstract a hydrogen atom from both the polar and nonpolar surfaces, we performed separate periodic DFT calculations on slabs of finite depth with the images separated by $\sim 20.0 \AA$. Optimized structures of the slabs with polar and nonpolar surfaces exposed are provided in the SI. The dissociation energy for removing a hydrogen atom was determined to be significantly greater from a polar surface site than from a nonpolar surface site, namely $4.3 \mathrm{eV}$ compared to $3.0 \mathrm{eV}$. While these dissociation energies are only approximate because of well-known shortcomings of these types of DFT calculations, particularly with the $\mathrm{PBE}$ functional, ${ }^{35}$ the relative dissociation energies are expected to be qualitatively correct. Moreover, previous experiments have indicated that a stable monolayer of hydrogen is formed on the O-terminated polar surface. ${ }^{27}$

The $\mathrm{H}$ sites are divided into bulk, subsurface, and surface sites. As mentioned above, all $\mathrm{O}(\mathrm{Zn})$ atoms with fewer than four nearestneighbor $\mathrm{Zn}(\mathrm{O})$ atoms are considered to be surface atoms. All $\mathrm{O}$ $(\mathrm{Zn})$ atoms within $2.0 \AA \AA$ of at least one surface $\mathrm{Zn}(\mathrm{O})$ atom belong to layer-2, while all $\mathrm{O}$ atoms within $2.0 \AA$ of at least one layer- $\mathrm{Zn}$ atom belong to the subsurface layer. The $\mathrm{H}$ sites on layer-2 are not considered because the slab calculations indicate that they are significantly higher in energy than the sites on the subsurface layer. All other $\mathrm{O}$ atoms that do not belong to the surface or subsurface layers, or to layer-2, constitute the bulk. Our model consists of 255 surface:up sites, 255 surface:down sites, 183 subsurface sites, and 675 bulk sites. Only the most stable $\mathrm{H}$ sites in the subsurface and bulk layers, along with two sites per surface $\mathrm{O}$ site, are considered as probable proton hopping sites.

Periodic DFT calculations were performed to identify the most stable $\mathrm{H}$ sites in the bulk and close to the surface. The bond-centered site parallel to the crystal $c$-axis (Figure $2 \mathrm{~A}$ ) was calculated to be the most stable site in the bulk, in agreement with previous calculations. ${ }^{36,37}$ In the subsurface layer, the most stable structure has the $\mathrm{H}$ pointing toward the surface at a bond-centered site (Figure $2 \mathrm{~B}$ ). This structure is $\sim 0.5 \mathrm{eV}$ higher in energy than the most stable surface site. On the surface, two plausible $\mathrm{H}$ sites, one beneath the surface and the other above the surface, were found to be stable (Figure 2C,D). The structure with $\mathrm{H}$ beneath the surface is $\sim 0.63 \mathrm{eV}$ higher in energy than the structure with $\mathrm{H}$ above the surface. To compare the stability of $\mathrm{H}$ at a site on the surface and at a site inside the bulk, we calculated the corresponding $\mathrm{H}$ atom addition energies as the difference in absolute energies of the slab or bulk with and without a $\mathrm{H}$ atom. The hydrogen affinities of a bulk site, a surface site, and a subsurface site were calculated to be $-2.4,-3.0$, and $-2.5 \mathrm{eV}$, respectively.

The microscopic rate constants for hopping between different types of neighboring sites were obtained using classical transition state theory, ${ }^{38}$ where the activation barriers were determined with the climbing image nudged elastic band method. ${ }^{39}$ Table 1 summarizes the activation energies for proton hopping between neighboring sites. The effective barrier for proton hopping between two bulk sites is calculated to be $\sim 0.7 \mathrm{eV}$. Similar estimates have been obtained from previous electronic structure calculations. ${ }^{37}$ Direct hopping between subsurface sites is associated with a significantly higher activation barrier, $1.2 \mathrm{eV}$, compared to hopping between a subsurface site and the nearest site on the surface, which was determined to be $0.3 \mathrm{eV}$. Note that the estimated rate constants should be considered to be only qualitatively accurate due to limitations of the PBE functional and the neglect of nuclear quantum effects such as zero-point energy and proton tunneling. The quantitative accuracy would be improved by using a hybrid functional and including such nuclear quantum effects beyond classical transition-state theory. Furthermore, as discussed above, the activation barrier for proton hopping from a bulk site to a subsurface site was treated as a parameter in this study. The calculation of this barrier with periodic DFT would require a significantly larger supercell that would be much more computationally expensive, 
particularly with a hybrid functional. For all of these reasons, the calculations presented herein should be viewed as providing a qualitative model that explains the experimental data rather than as quantitatively definitive results.

We performed KMC simulations of the proton diffusion among the available $\mathrm{H}$ sites. In these simulations, the immediate neighbor that accepts the proton is chosen randomly based on the cumulative distribution of the microscopic rate constants. After the proton has hopped from one site to another, the time is updated according to the following equation for the escape time: ${ }^{28}$

$$
\delta t_{i}=-\frac{1}{\sum_{j} k_{i j}} \log (u)
$$

where $u$ is a random number between 0 and 1 and $k_{i j}$ is the microscopic rate constant for jumps from site $i$ to site $j$. At the beginning of each KMC trajectory, a set of distinct reactive sites is chosen randomly. The diffusing proton starts either from a bulk site or a subsurface site with a uniform random distribution and hops to one of the neighboring sites randomly based on the hopping probabilities. This random walk continues until either the maximum total time is reached or the proton reacts. Calculations performed with $10^{5}, 10^{6}$, $10^{7}$, and $10^{8}$ random walks led to similar results in most cases, but to ensure convergence, the data reported herein correspond to simulations with $10^{7}$ random walks.

\section{ASSOCIATED CONTENT}

\section{S Supporting Information}

The Supporting Information is available free of charge on the ACS Publications website at DOI: 10.1021/acsnano.7b05009.

Computational details; plots of survival probabilities where the concentration of reactive sites is changed from $30 \%$ to $100 \%$; plot of survival probabilities where the activation barrier for bulk to bulk hopping is artificially increased to $0.8 \mathrm{eV}$; plot of survival probability with a certain distribution of oxygen vacancies at the interface of bulk and subsurface layers; histograms of walks for $E_{\mathrm{a}}^{\mathrm{B} \rightarrow S}$ $=0.5,0.6,0.9,1.0$, and $1.1 \mathrm{eV}$ (PDF)

Coordinates of bulk wurtzite, slabs with nonpolar and polar surfaces, $\mathrm{ZnO} \mathrm{NC}$, and all $\mathrm{H}$ sites; neighbor list in $\mathrm{ZnO} \mathrm{NC}$; rate constants for model lattice (TXT)

\section{AUTHOR INFORMATION}

\section{Corresponding Author}

*E-mail:shs3@illinois.edu.

\section{ORCID}

Sharon Hammes-Schiffer: 0000-0002-3782-6995

\section{Notes}

The authors declare no competing financial interest.

\section{ACKNOWLEDGMENTS}

This work was supported as part of the Argonne-Northwestern Solar Energy Research (ANSER) Center, an Energy Frontier Research Center funded by the U.S. Department of Energy, Office of Science, Office of Basic Energy Sciences. We thank Prof. James M. Mayer for helpful discussions.

\section{REFERENCES}

(1) Sauvage, F.; Nazeeruddin, M. K.; Gratzel, M. Metal-Oxide Nanoparticles for Dye-Sensitized Solar Cells. In Functional Metal Oxides, Ogale, S. B.; Venkatesan, T. V.; Blamire, M. G., Eds. WileyVCH: Weinheim, Germany, 2013; pp 341-383.

(2) Ameen, S.; Akhtar, M. S.; Song, M.; Shin, H. S. Metal Oxide Nanomaterials, Conducting Polymers and Their Nanocomposites for
Solar Energy. In Solar Cells - Research and Application Perspectives; Morales-Acevedo, A., Ed.; InTech: Rijeka, Croatia, 2013; pp 203-259.

(3) Furube, A.; Katoh, R; Hara, K. Electron Injection Dynamics in Dye-Sensitized Semiconductor Nanocrystalline Films. Surf. Sci. Rep. 2014, 69, 389-441.

(4) Pastore, M.; De Angelis, F. Modeling Materials and Processes in Dye-Sensitized Solar Cells: Understanding the Mechanism, Improving the Efficiency. In Multiscale Modelling of Organic and Hybrid Photovoltaics; Beljonne, D., Cornil, J., Eds.; Springer: Berlin, Heidelberg, Germany, 2014; pp 151-236.

(5) Akimov, A. V.; Neukirch, A. J.; Prezhdo, O. V. Theoretical Insights into Photoinduced Charge Transfer and Catalysis at Oxide Interfaces. Chem. Rev. 2013, 113, 4496-4565.

(6) O’Regan, B.; Gratzel, M. A Low-Cost, High-Efficiency Solar Cell Based on Dye-Sensitized Colloidal Tio2 Films. Nature 1991, 353, 737-740.

(7) Chen, X.; Mao, S. S. Titanium Dioxide Nanomaterials: Synthesis, Properties, Modifications, and Applications. Chem. Rev. 2007, 107, 2891-2959.

(8) Kumar, R.; Umar, A.; Kumar, G.; Nalwa, H. S.; Kumar, A.; Akhtar, M. S. Zinc Oxide Nanostructure-Based Dye-Sensitized Solar Cells. J. Mater. Sci. 2017, 52, 4743-4795.

(9) Goings, J. J.; Schimpf, A. M.; May, J. W.; Johns, R. W.; Gamelin, D. R.; Li, X. Theoretical Characterization of Conduction-Band Electrons in Photodoped and Aluminum-Doped Zinc Oxide (Azo) Quantum Dots. J. Phys. Chem. C 2014, 118, 26584-26590.

(10) Klahr, B.; Gimenez, S.; Fabregat-Santiago, F.; Hamann, T.; Bisquert, J. Water Oxidation at Hematite Photoelectrodes: The Role of Surface States. J. Am. Chem. Soc. 2012, 134, 4294-4302.

(11) Mandal, H.; Shyamal, S.; Hajra, P.; Samanta, B.; Fageria, P.; Pande, S.; Bhattacharya, C. Improved Photoelectrochemical Water Oxidation Using Wurtzite Zno Semiconductors Synthesized through Simple Chemical Bath Reaction. Electrochim. Acta 2014, 141, 294301.

(12) Sohn, Y.; Huang, W.; Taghipour, F. Recent Progress and Perspectives in the Photocatalytic Co2 Reduction of Ti-Oxide-Based Nanomaterials. Appl. Surf. Sci. 2017, 396, 1696-1711.

(13) Schrauben, J. N.; Hayoun, R.; Valdez, C. N.; Braten, M.; Fridley, L.; Mayer, J. M. Titanium and Zinc Oxide Nanoparticles Are ProtonCoupled Electron Transfer Agents. Science 2012, 336, 1298-1301.

(14) Braten, M. N.; Gamelin, D. R.; Mayer, J. M. Reaction Dynamics of Proton-Coupled Electron Transfer from Reduced Zno Nanocrystals. ACS Nano 2015, 9, 10258-10267.

(15) Knowles, K. E.; Tagliazucchi, M.; Malicki, M.; Swenson, N. K.; Weiss, E. A. Electron Transfer as a Probe of the Permeability of Organic Monolayers on the Surfaces of Colloidal Pbs Quantum Dots. J. Phys. Chem. C 2013, 117, 15849-15857.

(16) Van de Walle, C. G. Hydrogen as a Cause of Doping in Zinc Oxide. Phys. Rev. Lett. 2000, 85, 1012-1015.

(17) Van de Walle, C. G.; Neugebauer, J. Universal Alignment of Hydrogen Levels in Semiconductors, Insulators and Solutions. Nature 2003, 423, 626-628.

(18) Cox, S. F. J.; Davis, E. A.; Cottrell, S. P.; King, P. J. C.; Lord, J. S.; Gil, J. M.; Alberto, H. V.; Vilão, R. C.; Piroto Duarte, J.; Ayres de Campos, N.; Weidinger, A.; Lichti, R. L.; Irvine, S. J. C. Experimental Confirmation of the Predicted Shallow Donor Hydrogen State in Zinc Oxide. Phys. Rev. Lett. 2001, 86, 2601-2604.

(19) Hofmann, D. M.; Hofstaetter, A.; Leiter, F.; Zhou, H.; Henecker, F.; Meyer, B. K.; Orlinskii, S. B.; Schmidt, J.; Baranov, P. G. Hydrogen: A Relevant Shallow Donor in Zinc Oxide. Phys. Rev. Lett. 2002, 88, 045504.

(20) Shimomura, K.; Nishiyama, K.; Kadono, R. Electronic Structure of the Muonium Center as a Shallow Donor in Zno. Phys. Rev. Lett. 2002, 89, 255505.

(21) Kohiki, S.; Nishitani, M.; Wada, T.; Hirao, T. Enhanced Conductivity of Zinc Oxide Thin Films by Ion Implantation of Hydrogen Atoms. Appl. Phys. Lett. 1994, 64, 2876-2878. 
(22) Chianella, C.; Palombari, R.; Petricca, A. Electrochemical Hydrogen Doping of Zinc Oxide: A Study of the Oxide-Proton Conductor Interface. Electrochim. Acta 2006, 52, 369-372.

(23) Janotti, A.; Van de Walle, C. G. Fundamentals of Zinc Oxide as a Semiconductor. Rep. Prog. Phys. 2009, 72, 126501.

(24) Shi, G. A.; Stavola, M.; Pearton, S. J.; Thieme, M.; Lavrov, E. V.; Weber, J. Hydrogen Local Modes and Shallow Donors in Zno. Phys. Rev. B: Condens. Matter Mater. Phys. 2005, 72, 195211.

(25) Janotti, A.; Van de Walle, C. G. Hydrogen Multicentre Bonds. Nat. Mater. 2007, 6, 44-47.

(26) Bang, J.; Chang, K. J. Diffusion and Thermal Stability of Hydrogen in Zno. Appl. Phys. Lett. 2008, 92, 132109.

(27) Wöll, C. The Chemistry and Physics of Zinc Oxide Surfaces. Prog. Surf. Sci. 2007, 82, 55-120.

(28) Voter, A. F. Introduction to the Kinetic Monte Carlo Method. In Radiation Effects in Solids; Sickafus, K. E., Kotomin, E. A., Uberuaga, B. P., Eds.; Springer: Dordrecht, The Netherlands, 2007; pp 1-23.

(29) Koper, M. T. M.; van Santen, R. A.; Neurock, M. Theory and Modeling of Catalytic and Elctrocatalytic Reactions. In Catalysis and Electrocatalysis at Nanoparticle Surfaces; Wieckowski, A., Savinova, E. R., Vayenas, C. G., Eds.; CRC Press: Boca Raton, FL, 2003; pp 1-34.

(30) Valdez, C. N.; Schimpf, A. M.; Gamelin, D. R.; Mayer, J. M. Low Capping Group Surface Density on Zinc Oxide Nanocrystals. ACS Nano 2014, 8, 9463-9470.

(31) Dagdug, L.; Berezhkovskii, A. M.; Weiss, G. H. SmoluchowskiLike Theory for Trapping Kinetics in a Slab. Phys. A 2003, 318, 341346.

(32) Kakalios, J.; Street, R. A.; Jackson, W. B. Stretched-Exponential Relaxation Arising from Dispersive Diffusion of Hydrogen in Amorphous Silicon. Phys. Rev. Lett. 1987, 59, 1037-1040.

(33) Ghosh, S.; Castillo-Lora, J.; Soudackov, A. V.; Mayer, J. M.; Hammes-Schiffer, S. Theoretical Insights into Proton-Coupled Electron Transfer from a Photoreduced Zno Nanocrystal to an Organic Radical. Nano Lett. 2017, 17, 5762-5767.

(34) Valdez, C. N.; Schimpf, A. M.; Gamelin, D. R.; Mayer, J. M. Low Capping Group Surface Density on Zinc Oxide Nanocrystals. ACS Nano 2014, 8, 9463-9470.

(35) Hasnip, P. J.; Refson, K.; Probert, M. I. J.; Yates, J. R.; Clark, S. J.; Pickard, C. J. Density Functional Theory in the Solid State. Philos. Trans. R. Soc., A 2014, 372, 20130270.

(36) Limpijumnong, S.; Zhang, S. B. Resolving Hydrogen Binding Sites by Pressure-a First-Principles Prediction for Zno. Appl. Phys. Lett. 2005, 86, 151910.

(37) Wardle, M. G.; Goss, J. P.; Briddon, P. R. First-Principles Study of the Diffusion of Hydrogen in Zno. Phys. Rev. Lett. 2006, 96, 205504.

(38) Eyring, H. The Activated Complex in Chemical Reactions. J. Chem. Phys. 1935, 3, 107-115.

(39) Henkelman, G.; Uberuaga, B. P.; Jónsson, H. A Climbing Image Nudged Elastic Band Method for Finding Saddle Points and Minimum Energy Paths. J. Chem. Phys. 2000, 113, 9901-9904. 